\title{
Nachdenken im Kinosessel? Bioethische Reflexion durch Filme als eine neue Möglichkeit der Diskussion von Standpunkten und Betroffenheit
}

\author{
Sabine Wöhlke • Solveig Lena Hansen · Silke Schicktanz
}

Online publiziert: 23. Januar 2015

(C) Springer-Verlag Berlin Heidelberg 2014

Die Filmkomödie Ziemlich beste Freunde ${ }^{1}$ beruht auf der Autobiografie eines Tetraplegikers. Im Fokus steht die Freundschaft zwischen dem behinderten Phillipe und seinem langjährigen Pflegehelfer, der ihm mit seiner unkonventionellen Art neuen Lebensmut gibt. Dieser Film, der von Kritikern als der passende Spaßfilm zur heutigen „Problem-Zeit“" bezeichnet wird, besticht durch seinen ungewöhnlichen Humor, da er „eher unüblich sei, weil man über Behinderte keine Witze macht oder die Leute, die über Behinderte Witze machen, in aller Regel abstoßend sind“" [30].

Solche filmischen Annäherungen an existierende bioethische Probleme, wie in diesem Beispiel des intuitiven und würdevollen Umgangs mit körperlich stark eingeschränkten Personen, setzen neue Impulse für eine kritische Auseinandersetzung mit gegenwärtigen Reha- und Versorgungskonzepten.

Sie bieten mehr als nur eine „Illustration“ von Chancen und Herausforderungen einer Technik, sondern sind vielmehr eine eigene Wissensform unserer kulturellen und kollektiven Identität [11]. Das Beispiel Ziemlich beste Freunde verdeutlicht, dass Filme mit bioethischem Potential für eine Auseinandersetzung mit den kontroversen Folgen einer durch Technisierung veränderten Lebenswelt dienen können. Im Fernsehen und im Kino greifen sie Alltagserfahrungen und -konflikte auf, die sie wiederum dramatisieren, abstrahieren und zu „kleinen“ Geschichten verarbeiten [4]. Auch wenn manche dieser filmischen Szenarien als überzeichnet anzusehen sind, bieten die erzählten Geschichten eine angereicherte Darstellung des Verhältnisses zwischen dem System der Biomedizin und den darin agierenden Individuen.

Filme nehmen aufgrund ihrer narrativen und ästhetischen Struktur einen jeweils sehr spezifischen Standpunkt zur sozialen Realität, der lebensweltlichen Orientierung und der

${ }^{1}$ Ziemlich beste Freunde, F (2011).

Dr. des. S. Wöhlke $(\bowtie) \cdot$ S. L. Hansen, M.A. · Prof. Dr. S. Schicktanz Institut für Ethik und Geschichte der Medizin, Universitätsmedizin Göttingen, Humboldtallee 36, 37073 Göttingen, Deutschland

E-Mail: sabine.woehlke@medizin.uni-goettingen.de 
individuellen und kollektiven Identitätsbildung ein. Diese können die Perspektiven aus dem ethischen Fachdiskurs bestätigen, neu verhandeln oder ihnen auch widersprechen. Einzelne Handlungen der Figuren und ganze Lebensentwürfe werden für den Zuschauer manchmal mehr, manchmal weniger eindeutig als moralisch, unmoralisch oder auch amoralisch dargestellt. Der moralische Standpunkt des Pflegers in Ziemlich beste Freunde ist beispielsweise, dass der (wohlhabende) Tetraplegiker Phillipe keinen besonders schonenden Umgang auf der sozialen und kommunikativen Ebene braucht. Der Pfleger selbst wiederum hat keine Ausbildung; ihn qualifiziert aus Phillipes Sicht vielmehr seine Unbedarftheit, sein ehrlich artikulierter Widerwille und sein fehlendes Mitleid. Indem gerade diese Eigenschaften als tugendhaft dargestellt werden und der Pfleger durch sie moralisch handelt, nimmt der Film eine sehr spezielle ethische Perspektive auf das Phänomen der Behinderung ein, die mit den gängigen sozialen Tabus bricht und damit unseren eingeübten Umgang hinterfragt.

Hier zeigt sich auch, dass Filme medizinische Techniken oder Handlungen sowie Krankheiten selten einfach nur „darstellen“ $[18,29]$. Vielmehr kontextualisieren sie diese im Sozialen, indem die Figuren affiziert werden: Sie leiden, erlangen Hilfe oder werden gegenüber anderen Menschen benachteiligt. Durch diesen personenbezogenen Zugang über Filme kann der akademische, meist abstrakte Diskurs für die (oft unterschiedlichen) Perspektiven von Betroffenen geöffnet werden [16]. Einerseits thematisieren Filme die Betroffenheit der Figuren, andererseits wird ein „,betroffenes“ Publikum angesprochen. Der Begriff der „Betroffenheit“ hat allerdings gerade beim Film zwei Dimensionen: zum einen die emotionale Reaktion auf die visuell geschilderten Ereignisse, die sich in Reaktionen wie Mitgefühl, Besorgtheit bis hin zu Bestürztheit äußern können. In diesem Sinne muss also keine objektive Beziehung zum Sachverhalt vorliegen, der subjektive Gefühlszustand reicht für eine „Betroffenheit“ bereits aus. Zum anderen meint Betroffenheit eine objektive Kausalbeziehung, bei der eine oder mehrere Personen durch ein Ereignis oder Sachverhalt in Mitleidenschaft gezogen werden. „Objektiv“ meint hier, dass die besagte Beziehung unabhängig vom subjektiven Bewusstsein der involvierten Person(en) selbst ist. So kann eine Person z. B. von radioaktiver Strahlung betroffen sein, ohne sich betroffen zu fühlen oder ihre Betroffenheit zu bemerken [23]. Diese Form des Betroffenendiskurses beinhaltet einen Perspektivenpluralismus, der nicht allein auf Meinungsdifferenzen, sondern auf unterschiedliche Formen des Rollenverständnisses, der Erfahrung mit dem medizinischen System oder auch moralisch-religiösen Grundhaltungen zurückzuführen ist [21].

\section{Inhaltliche Erwägungen zu Filmen mit bioethischem Potenzial}

Wir gehen davon aus, dass bioethische Urteile stets gemischte Urteile sind (vgl. [7], S. 26 f.), da sie sich aus deskriptiven Teilaussagen (Was ist bereits möglich? Wie funktioniert das? In welches gesellschaftliche und kulturelle Setting ist Technik eingebettet?), prognostischen Aussagen (Welche Folgen könnte eine Handlung nach sich ziehen? Welche zukünftigen Möglichkeiten bietet eine Technik?) und präskriptiven Prämissen (Ist die Handlung moralisch vertretbar? Gibt es Bedingungen, die diese Vertretbarkeit beschränken? Ist eine solche Zukunft wünschenswert?) zusammensetzen. Filme können auf allen drei Ebenen dieser Urteilsbildung ansetzen und es ist beim Einsatz eines Filmes für bioethische Reflexion äußerst hilfreich, deutlich zu machen, welche Ebene in den Blick genommen wird. 
Anthropologische Annahmen, beispielsweise über den Körper, können einen wichtigen und auch kritischen Teil der Deskription darstellen. Derartiges anthropologisches Wissen ist zumeist symbolisch und vor allem relativistisch, weil es „lokal“ verortet, d. h. kulturell unterschiedlich ist. Biomedizinisches Wissen über den Körper wird hingegen oft als universal, objektivistisch, materialistisch und reduktionistisch dargestellt. Während der klinische biomedizinische Blick auf den Körper diesen in immer kleinere unsichtbare Teilchen fragmentiert, favorisiert die medizinanthropologische Sicht stärker auf einen mehrdimensionalen Körper. Dabei wird von einem repräsentativen „,sozialen“ Körper (Identität), den Kräften des „politischen“ Körpers (Macht) und einem „persönlichen“ existenziellen Körper (Leib/Körper) ausgegangen [20]. Diese anthropologische Perspektive dient somit auch als Kritik einer bestimmten, nur vermeintlich objektiven Deskription, wenn z. B. aufgezeigt wird, dass diese Beschreibungen selbst wiederum in Machtstrukturen eingebettet sind. Gerade Filme können als Teil einer solchen Anthropologie genutzt werden. Arno Görgen und Rudolf T. Inderst zeigen in ihrem Beitrag „Land der lebenden Toten“ im vorliegenden Heft diese Differenzierungen auf, indem sie die Ausprägungen und Folgen des Diskurses um Körper der 1960er Jahre mithilfe der Fernsehserie The Walking Dead ${ }^{2}$ analysieren. Die Serie bedient sich der Form einer Negation biologischer Gewissheiten, um den Zerfall von personalen, familiären und gesellschaftlichen Gewissheiten und Wertestrukturen zu thematisieren. Am deutlichsten, so die Autoren, wird diese Verunsicherung angesichts des Umganges mit dem Tod sichtbar.

Doch nicht nur für die anthropologische Perspektive, sondern auch für die anderen Teilaussagen können Filme als visuell ansprechende, aber auch emotionale Konkretisierung bioethischer Themen genutzt werden, um sich zunächst ausgehend vom filmischen Impuls deskriptiv über Möglichkeiten von Medizin und Technologie zu informieren [10]. Als Form kultureller Technikfolgenabschätzung beinhalten Filme außerdem, ähnlich wie Literatur, prognostische Aussagen über die zukünftige Machbarkeit und gesellschaftliche Veränderungen von Techniken (vgl. [9]). Hinter vermeintlich nüchternen Konflikten über Technikszenarien lassen sich häufig grundlegende Wertekonflikte auffinden, die auf die dritte Art von Teilaussagen, nämlich Präskriptionen verweisen. Hier können Filme einerseits Szenarien als Impuls für die Frage darstellen, ob eine bestimmte, filmisch dargestellte Handlung ethisch vertretbar ist oder ob es bestimmte Bedingungen gibt, die eine Beschränkung der Handlungsfreiheit aus ethischen Gründen legitimieren. Diese Antwort muss anschließend nach der ethischen Vertretbarkeit beurteilt werden, um die präskriptive Dimension solcher Techniken zu bewerten [13]. Gerade Filme können aufgrund ihrer komplexen narrativen Strukturen dazu beitragen, diese ethische Vertretbarkeit zu diskutieren, indem man verschiedene, auch konkurrierende Theorien auf die Praxis ,anwendet“. Dabei muss dem Film noch kein eigener ethischer Standpunkt zugewiesen werden, sondern alternative Rezeptionen (affirmativ, kritisch, ironisch etc.) können als Ausgangspunkt für einen solchen Dialog zwischen Theorien und Anwendung dienen. Leitend hierfür sind ethisch motivierte Fragen nach der jeweiligen ästhetisierten Perspektive: Wessen Perspektive wird gezeigt, wessen Position wird verteidigt, welche Konsequenzen haben die imaginierten Technologien.

Zudem erscheint es uns ethisch relevant, dass Filme insofern der realen komplexen Alltagssituation sehr nahe kommen, weil soziale Abhängigkeiten, Verletzlichkeiten und Emotionen in Grenzsituationen thematisiert werden, wie sie in abstrakten Beschreibungen

${ }^{2}$ The Walking Dead, USA (2010). 
selten möglich sind. So lädt beispielsweise The Island ${ }^{3}$ dazu ein, sich in die Rolle eines Klons hineinzuversetzen und Empathie für dessen Perspektive auf gerechte und lebenswerte Bedingungen zu entwickeln. Dies wiederum regt die ethische Diskussion an, über die Konstruktion des Klons als abhängiges, unselbstständiges Monstrum nachzudenken. Auf einer reflexiven Ebene geht es jedoch nicht nur um eine Perspektive des fiktiven Klons, sondern vielmehr um die Perspektivenübernahme für Personen mit geringem Handlungsspielraum. Der Film verhandelt daher nicht nur Biotechnologien, sondern auch den Informations- und Agitationsraum von besonders vulnerablen Figuren. Er bietet damit viel kritisches Potential, über mangelnde Aufklärung, Abhängigkeitsverhältnisse oder ungleiche strukturelle Rahmenbedingungen, wie sie in unserer Gesellschaft für andere ausgegrenzte Personen existieren (z. B. Flüchtlinge, Migranten), nachzudenken und die Verbindung zwischen verschiedenen Diskursen aufzuzeigen (vgl. allgemein [9]). Solveig Lena Hansen und Sabine Wöhlke zeigen den Mehrwert eines solchen Zugangs in ihrem Beitrag „Institutionalisierte Uninformiertheit als Bedingung von Vulnerabilität beim Klonen und Organspende in Never Let $\mathrm{Me} \mathrm{Go}^{4 \text { " }}$ auf. Sie analysieren die moralische Position des Filmes nicht nur in Bezug auf die Techniken (Klonen/Organtransplantation) selbst, sondern gehen der Frage nach, welcher Standpunkt sich in Bezug auf soziale Mobilität, Vulnerabilität und Abhängigkeit im Film selbst bzw. durch seine Rezeption entwickeln lässt.

Fallbeispiele ohne affizierte Figuren neigen in der Tendenz durch ihre Abstraktion oft dazu, dass ein Problem als unlösbares Dilemma präsentiert wird und an diesem „nur“ gegensätzliche ethische Theorien diskutiert bzw. veranschaulicht werden. Viele Filme weisen zwar in sich Ambivalenz und unaufgelöste Dilemmata auf, jedoch lassen sich diese (und das unterscheidet sie häufig von Fallbeispielen) als inhärente Sozial- aber auch Medizinkritik wieder an ihren Entstehungskontext zurückbinden [24]. Schon Filmklassiker wie Soylent Green ${ }^{5}$ oder Blade Runner ${ }^{6}$ versuchten in den 1970er und 1980er Jahren, aktuelle Gesellschaftsentwicklungen in eine filmische Zukunft zu projizieren, um so Kritik an der Verbindung von sozialem Raum, Politik, Wirtschaft und auch Wissenschaft zu üben [14]. Während der rein analytische Blick dazu neigt, eine solche Kontextbezogenheit des Fallbeispiels selbst außer Acht zu lassen, bettet die Analyse und Interpretation fiktionaler Szenarien diese häufig wieder an die „Realität“ zurück, z. B. indem gefragt wird, welche konkreten Werte und praktischen sozialen Techniken über die Themen der Biomedizin implizit maßgeblich verhandelt werden [9].

\section{Methodische Herangehensweisen an Filme mit bioethischem Potenzial}

Neben diesen grundsätzlichen Überlegungen zum kulturtheoretischen Reflexionspotenzial von Filmen für die Forschung stellt die medizinethische Lehre bisher die zugänglichste methodische Schnittstelle von Bioethik und Film dar [28]. Ein grundlegendes didaktisches Ziel besteht zunächst darin, Studierenden die drei Dimensionen (deskriptiv, prognostisch, präskriptiv) des bioethischen Urteils zu vermitteln und ihre Argumentationskompetenz

\footnotetext{
${ }^{3}$ The Island, USA (2005).

${ }^{4}$ Never Let Me Go, USA (2011).

${ }^{5}$ Soylent Green, USA (1973).

${ }^{6}$ Blade Runner, USA (1982).
} 
dahingehend zu schärfen, dass sie fremde wie eigene Argumente kritisch prüfen [6]. Gerade Filme eignen sich dabei aufgrund ihrer Kurzlebigkeit und Visualität, sie im o. g. Sinne auch in der Lehre einzusetzen. Filminhalt und Bedeutung sind prinzipiell das Resultat eines differenzierten Zusammenwirkens verschiedener, während der Rezeption meist unbewusst wahrgenommener Faktoren. Die filmische Aussage entsteht erst über die Leinwandprojektion hinaus in der Wahrnehmung durch das Publikum und ist damit nur zum Teil auf den Film selbst zurückzuführen. Die rezipierte Botschaft als Summe filminterner und -externer Einflussfaktoren ist eine durch individuelle, situative und historisch-gesellschaftliche Variablen beeinflusste Konstruktion des Zuschauers ([15], S. 14). Filme können als Schnittstelle zwischen dem Wechselspiel der gezeigten Bilder und der Wahrnehmung des Körpers [8] fungieren.

Insbesondere der Beitrag von Katja Kailer, „Ethische Urteilsbildung mittels Spielfilme?“ zeigt an einem der bioethischen „Filmklassiker“, nämlich GATTACA auf, dass Modelle der didaktischen Urteilsbildung auf das fiktionale Medium des Spielfilms übertragen werden können [25].

Das Beispiel zeigt, dass Filme letztlich für die medizinethische Lehre als Ergänzung zum schlüssigen Argumentieren zu verstehen sind, indem sie dieses stimulieren und eine rein abstrakte Bewertung mit einem ansprechenden Fallbeispiel ergänzen [2]. Methodisch können sie mit einer Alltagsanschaulichkeit zur Entdeckung von bioethischen Konflikten führen und neugierig machen, jedoch können sie die rationale bioethische Diskussion nicht ersetzen. Gerade die implizite moralische Bewertung des Films oder auch eine gänzlich fehlende moralische Position kann ebenso Anlass für eine kritisch-erweiternde Analyse eines Themas geben.

In eine solche Analyse sind allerdings nicht nur die Aussagen des Films, sondern auch sein Kontext, also die Entstehungszeit, Aussagen der Produzierenden sowie die Rezeption mit einzubeziehen, um kritisch herauszuarbeiten, dass auch Filme ihren Standpunkt im Kontext ihrer Zeit entwickeln [27]. Auf beide Aspekte (Ergänzung klassischer didaktischer Ethik-Modelle sowie Kontextanalysen) gehen bio- und medizinethische Lehrbücher bisher allerdings kaum ein (z. B. [26]).

Neben Spielfilmen, deren Vorteil in ihrer Fiktionalität besteht, lassen sich auch Dokumentarfilme für bioethische Diskurse nutzen. Ein Dokumentarfilm lässt sich im Wesentlichen an einer strengen Befolgung einer naturalistischen Perspektive, die eine Verknüpfung zwischen bewegtem Bild und gesprochenem Wort fordert, erkennen. Der Film will eine objektive „Wirklichkeit“ einfangen, indem der Filmemacher nur real existierende Personen in realen Situationen filmt. So verstehen sich Dokumentarfilmer zumeist als Beobachter. Der Fokus liegt auf Alltagserfahrungen, die durch das „Soziale“ definiert werden. Hier zeigt sich, dass im Grenzbereich zwischen Fiktion und Realität kulturelle Unterschiede zusammenfließen, die ethnischer, alters- und geschlechtsspezifischer Natur sein können und die Produktion und Rezeption von Filmen als einer ,sozialen Praxis“ in einer bestimmten Zeit beeinflussen: im Zusammenwirken eines „Erfahrungspotenzials“ im Umgang mit medialen Produktionen und eines ,gesellschaftlichen Ortes“, der von verschiedenen, sich immer wieder verändernden Diskursen durchdrungen ist. Der Dokumentarfilm nimmt die wirkliche Welt als Ausgangspunkt, er bedient sich einer Ästhetik der Objektivität und einer technischen Apparatur, die wahre Aussagen (Bilder) über die Welt hervorbringt ([5], S. 419).

In methodischer Hinsicht geht insbesondere der Aufsatz von Sayani Mitra und Solveig Lena Hansen mit dem programmatischen Titel „Auf der anderen Seite der Kamera“ einen 
neuen Weg, indem anhand des Themas der Leihmutterschaft die Perspektive der Filmschaffenden selbst durch die Methode des Experteninterviews [1] in den Vordergrund gerückt wird. Dabei zeigt sich am Beispiel des Dokumentarfilms, dass diesem Medium durchaus ein moralischer Standpunkt eingeschrieben ist.

\section{Themen einer ethischen Auseinandersetzung mit Filmen}

Schwerpunktmäßig thematisiert dieses Heft zwei Felder, nämlich Anfang und Ende des menschlichen Lebens [3, 19]. Diese beiden Bereiche berühren durch die Phänomene der Reproduktion und des Alterns sowie Todes nicht nur zwei prominente Themen der Bioethik, sondern auch anthropologische Konstanten [17]. Geboren werden und alt werden bzw. sterben sind Phänomene, die Individuen, Familien, Kollektive und deren Institutionen fundamental betreffen, und werden deshalb auch im vorliegenden Themenheft behandelt.

Zudem zeigen sich gerade hier soziale und individuelle Verschränkungen ethischer Konflikte: Die Frage, ob Personen gezeugt oder nicht gezeugt werden, ob sie am Leben bleiben oder wie mit ihren Alterserscheinungen sowie mit Sterben und ihrem Tod umzugehen ist, sind Aspekte, die die individuelle Betroffenheit von einem Phänomen wieder an die kollektive Dimension zurückbinden.

Deutlich wird dies einerseits am Thema Fortpflanzung, wie es Tobias Eichinger in seinem Beitrag „Die Inszenierung unserer reproduktiven Zukunft“ analysiert. Hier steht die Frage im Fokus, wie eine bestimmte Reproduktionstechnik, in diesem Fall die Leihmutterschaft, vor einer sozialen Ordnung zu sehen ist. Des Weiteren stellt der Artikel durch die Positionierung in der narrativen Ethik eben dieses Thema in einen hermeneutischen Zugang zu bioethischen Themen- und Problemfeldern [12].

Anderseits werfen Altern und Sterben nicht zuletzt durch den Demografischen Wandel zahlreiche bioethische Fragen auf. Filme wie Halt auffreier Strecke thematisieren das Sterben nicht mehr nur als Handlungsnebenstrang, sondern rücken das Thema in den Mittelpunkt. Ein Arzt hat dem Patienten mitgeteilt, dass bei ihm ein Tumor festgestellt wurde, seine Zeit ist ab jetzt bemessen. Unweigerlich stellen sich für den Patienten Fragen nach dem warum ich und warum jetzt? Er lässt Frau und Kinder, Eltern, Freunde und Nachbarn zurück, alles Personen, die eine wichtige Rolle in seinem Leben spielen. Mit großer Sensibilität und Aufrichtigkeit zeigt der Film den Umgang mit dem stetigen Verfall der sterbenden Hauptfigur und die Überforderung der Familie mit dieser Situation, und verdeutlicht, dass Sterben sowie Fragen zu Entscheidungen am Lebensende nicht nur im Zusammenhang mit Alter gesehen werden sollten.

Alter und Altern stellt alle unmittelbar Beteiligten ebenso wie ihre Umgebung auch unabhängig vom Sterbeprozess aufgrund von Krankheiten wie Demenz vor neue ethische und gesellschaftliche Herausforderungen. Der Beitrag von Mark Schweda und Lisa Frebel greift das Thema Altern mit der Frage „Wie ist es, dement zu sein?“ auf. Die beiden Autor/innen zeigen nicht nur durch ein bestimmtes Krankheitsbild alternder Menschen ein weiteres Themenfeld auf, sondern eröffnen eine Verstehensdimension bioethisch relevanter Phänomene, die in ihrem Fall besonders die Betroffenenperspektive fokussiert.

Nicht zuletzt zeigen diese beiden Artikel, dass Filme nicht nur eine inhaltliche Perspektive auf normative Fragestellungen anbieten, sondern auch eine epistemische Perspektive

${ }^{7}$ Halt auf freier Strecke, D (2011). 
zu Biotechnologien und Medizin bereithalten: Auf der Darstellungs- und Wahrnehmungsebene stellt sich zunächst einmal die Frage, was in den Filmen eigentlich warum gezeigt wird und welches Wissen dadurch vermittelt wird. Hiermit lässt sich die Frage der epistemischen Gerechtigkeit [22] auf den Film übertragen, wenn gefragt wird, wessen Perspektive in einem (fiktionalen) Diskurs Gewicht bekommt. Aus dieser Perspektive gibt es nicht nur bioethische Themen als Inhalte des Films, über die die Figuren diskutieren und die sie handelnd umsetzen; vielmehr wird der Film selbst zu einem kulturellen und fiktionalen Anwendungsfeld und damit zu einer technischen Handlung, deren Ergebnisse eine je eigene Darstellungsweise beinhalten.

\section{Literatur}

1. Bogner A, Littig B, Menz W (2009) Experteninterviews. Theorien, Methoden, Anwendungsfelder. Springer, Wiesbaden

2. Burstein M (2009) The thanatoria of Soylent Green: on reconciling the good life with the good death. In: Shapshay S (Hrsg) Bioethics at the movies. John Hopkins University Press, Baltimore, S 275-296

3. Clarke A, Mamo L, Fosket JR, Fishman JR, Shim JK (2010) Biomedicalization. Technoscience, health, and illness in the U.S. Duke University Press, Durham London

4. Coldry N, Hepp A, Krotz F (2009) Media events in global age. Routledge Chapman \& Hall, London

5. Denzin NK (2002) Reading Film - Filme und Videos als sozialwissenschaftliches Erfahrungsmaterial. In: Flick U, von Kardorff E, Steinke I (Hrsg) Qualitative Forschung. Ein Handbuch. Rowohlt, Hamburg, S 416-429

6. Dietrich J (1999) Wissenschaftsethik und Ethikunterricht: Ergebnisse des Projekts „Schule Ethik Technologie" (SET). Ethik Unterr 4:35-41

7. Düwell M (2008) Bioethik. Methoden, Theorien und Bereiche. Metzler, Stuttgart Weimar

8. Elsaesser T, Hagener T (2010) Film theory. An introduction through the senses. Routlegde, New York

9. Hansen SL (2014) Alterität als kulturelle Herausforderung des Klonens. Eine Rekonstruktion bioethischer und literarischer Verhandlungen. Dissertation, Georg-August-Universität Göttingen (eingereicht Oktober 2014)

10. Hansen SL, Wöhlke S (2014) Movies, ready for an ethical debate? Soz Tech 1:5-7

11. Hepp A (2010) Cultural Studies und Medienanalyse. Eine Einführung, 3. Aufl. VS Verlag für Sozialwissenschaften, Wiesbaden

12. Joisten K (2009) Narrative Ethik. Das Gute und das Böse erzählen. Oldenbourg Akademieverlag, Berlin

13. Köchy K (2008) Kontextualistische Bioethik - Zur Rolle von biowissenschaftlichen Fakten bei bioethischen Fragen. In: Zichy M, Grimm H (Hrsg) Praxis in der Ethik. Zur Methodenreflexion in der angewandten Moralphilosophie. de Gruyter, Berlin, S 153-184

14. Koebner T (2003) Filmgenres. Science Fiction. Reclam, Stuttgart

15. Korte H (2001) Einführung in die systematische Filmanalyse. Erich Schmidt Verlag, Berlin

16. Lepa S (2010) Jenseits des Films. Kritisch-realistische Rekonstruktion von Filmverstehen und Filmreflexion. VS Verlag für Sozialwissenschaften, Wiesbaden

17. Litch M (2002) Philosophy through film. Routledge, London

18. Livingston P (2006) The very idea of film as philosophy. J Aesthet Art Crit 64(1):11-18

19. Quante M (2002) Personales Leben und menschlicher Tod. Suhrkamp, Frankfurt a. M.

20. Scheper-Hughes N (1994) Embodied knowledge: thinking with the body in critical medical anthropology. In: Borofsky R (Hrsg) Assessing cultural anthropology. McGraw-Hill, New York, S 229-239

21. Schicktanz S (2009) Zum Stellenwert von Betroffenheit, Öffentlichkeit und Deliberation im empirical turn der Medizinethik. Ethik Med 21(3):223-234

22. Schicktanz S (2012) Epistemische Gerechtigkeit. Sozialempirie und Perspektivenpluralismus in der Angewandten Ethik. Dtsch Z Philos 60(2):269-283

23. Schicktanz S, Schweda M, Franzen M (2008) 'In a completely different light'? - the role of being affected for epistemic perspectives and moral attitudes of patients, relatives and lay people. Med Health Care Philos 11:57-72

24. Schmidt K (2008) Spielfilme als Sensibilisierung für medizinethische Themenfelder. In: Schmidt K, Maio G, Wulff HJ (Hrsg) Schwierige Entscheidungen. Krankheit, Medizin und Ethik im Film. Haag + Herchen, Frankfurt a. M., S 29-38 
25. Shapshay $\mathrm{S}$ (2009) Lifting the genetic veil of ignorance: is there anything really unjust about Gattacan society? In: Shapshay S (Hrsg) Bioethics at the movies. John Hopkins University Press, Baltimore, S $87-102$

26. Thies C (2011) Medienethik. In: Stoecker R, Neuhäuser C, Raters ML (Hrsg) Handbuch Angewandte Ethik. Metzler, Stuttgart, S 206-209

27. Winter R (2006) Die Filmtheorie und die Herausforderung durch den „perversen Zuschauer“. Kontexte, Dekonstruktionen und Interpretationen. In: Mai M, Winter R (Hrsg) Das Kino der Gesellschaft - die Gesellschaft des Kinos: Interdisziplinäre Positionen, Analysen und Zugänge. Herbert von Halem Verlag, Köln, S 79-94

28. Wöhlke S, Schicktanz S (2010) Movies as teaching material - ethical issues in organ transplantation. In: Schicktanz S, Wiesemann C, Wöhlke S (Hrsg) Teaching ethics in organ transplantation and tissue donation. Cases and movies. Universitätsverlag Göttingen, Göttingen, S 67-76

29. Wulff HJ (2003) Empathie als Dimension des Filmverstehens. Montage AV Z Theor Gesch Audiov Kommun 12(1):136-161

30. Wulff M (2012) Männer, die mit den Ohren Sex haben. Resource Document. Die Welt. Axel Springer AG. 5. Januar 2012. http://www.welt.de/kultur/kino/article13797604/Maenner-die-mit-den-Ohren-Sexhaben.html. Zugegriffen: 13. Dez. 2014 